NASA Contractor Report 181861

ICASE Report No. 89-38

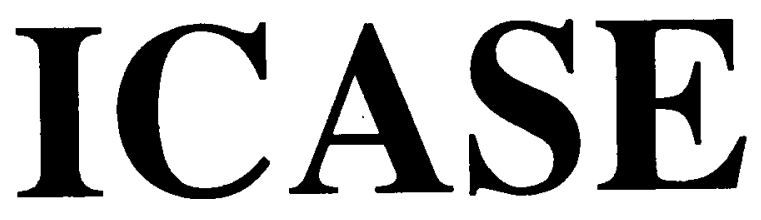

\title{
ABSOLUTE/CONVECTIVE INSTABILITIES AND THE CONVECTIVE MACH NUMBER IN A COMPRESSIBLE MIXING LAYER
}

\author{
T. L. Jackson \\ C. E. Grosch
}

(NASA-CR-181861) ABSOL OTE/CONVECTIVE

I USTABILITIES AYD THE CONVECTIVE AACH NOHBE

In a COAPRESSIBLE HIXING LAYBR Final Beport (ICASE) $24 p$
CSCL 20D
$489-27113$

Unclas

0224110

Contract No. NAS1-18605

June 1989

Institute for Computer Applications in Science and Engineering

NASA Langley Research Center

Hampton, Virginia 23665-5225

Operated by the Universities Space Research Association

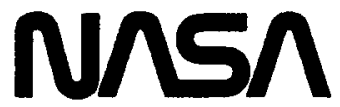

National Aeronautics and

Space Administration

Langley Research Center

Hampton, Virginia 23665-5225 


\title{
ABSOLUTE/CONVECTIVE INSTABILITIES AND THE CONVECTIVE MACH NUMBER IN A COMPRESSIBLE MIXING LAYER
}

\author{
T. L. Jackson \\ Department of Mathematics and Statistics \\ Old Dominion University \\ Norfolk, Virginia 23529 \\ C. E. Grosch \\ Department of Oceanography and \\ Department of Computer Science \\ Old Dominion University \\ Norfolk, Virginia 23529
}

\begin{abstract}
In this paper we consider two aspects of the stability of a compressible mixing layer: Absolute/Convective instability and the convective Mach number. We show that, for Mach numbers less than one, the compressible mixing layer is convectively unstable unless there is an appreciable amount of backflow. We also present a rigorous derivation of a convective Mach number based on linear stability theory for the flow of a multi-species gas in a mixing layer. Our result is compared with the heuristic definitions of others and to selected experimental results.
\end{abstract}

This work was supported by the National Aeronautics and Space Administration under NASA Contract NAS1-18605 while the authors were in residence at the Institute for Computer Applications in Science and Engineening, NASA Langley Research Center, Hampton, VA 23665 . 
1. Introduction. In the past few years there has been an increasing interest in the stability of compressible mixing layers. This results, in part, from the projected use of the scramjet engine for the propulsion of hypersonic aircraft. Knowledge of the stability of the mixing layers in the combustor may allow one to control the downstream evolution of the flow.

Both temporal and spatial stability calculations have recently been carried out for compressible mixing layers. If the flow is absolutely unstable its stability is properly described by temporal theory, but if it is convectively unstable spatial theory is appropriate. It is known that the incompressible mixing layer is generally convectively unstable but that a sufficiently large backflow can make it absolutely unstable (Huerre and Monkewitz, 1985). Such information is not available for the compressible mixing layer for which temperature effects are important.

The first part of the work reported here is to determine whether a compressible mixing layer is absolutely or convectively unstable. The calculations are presented in section 2 and results are given as a function of the Mach number of the fast stream and the velocity and temperature ratios of the streams.

An additional reason for the renewed interest in the stability of compressible mixing layers is that a number of experiments (e.g., Brown and Roshko, 1974; Chinzei, Masuya, Komuro, Murakami, and Kudou, 1986; and Papamoschou and Roshko, 1986, 1988) and numerical simulations (e.g., Guirguis, 1988; Lele, 1989; Sandham and Reynolds, 1989; and Mukunda, Sekar, Carpenter, Drummond, and Kumar, 1989) have shown that the mixing layer becomes less unstable with increasing Mach number. In order to correlate the experimental results, a number of experimentalists have used a heuristically defined "convective Mach number". This idea, first introduced by Bogdanoff (1983) for compressible flows, has resulted in several different definitions of a convective Mach number. In addition, when a mixing layer is formed between two different gases some definitions will yield different values for the convective Mach number in the two gases. It is not obvious which value is the proper one to use in the correlation, or whether some average of the two is appropriate.

We have recently carried out extensive spatial stability calculations for a compressible mixing layer (Jackson and Grosch, 1989a,b). Our results suggested a way to rigorously derive from linear stability theory a single convective Mach number for a compressible mixing layer for both a single species gas and a multi-species gas. In section 3 we present that derivation and discuss its application. We also present a comparison with selected experimental results.

2. Absolute/Convective Instabilities. The concept of absolute and convective instabilities was introduced by Briggs (1964) in the context of plasma instability. The same idea was put forward independently by Gaster $(1968,1981)$ within the context of the classical theory of hydrodynamic stability based on the Orr-Sommerfeld equation. Very recently these concepts have been applied to classify the instabilities of incompressible flows; for example, the mixing layer (Huerre and Monkewitz, 1985); capillary instabilities 
(Leib and Goldstein, 1986a,b); and wakes (Hultgren and Aggarwal, 1987; Monkewitz, 1988). A review of these concepts can be found in Huerre (1987).

A flow is said to be absolutely unstable if the response to an impulse in space and time is unbounded everywhere in space for large time. On the other hand, if the response decays to zero everywhere in space for large enough time, the flow is said to be convectively unstable. In this latter case the response to the impulse is a wave packet propagating downstream from the source with the waves forming the packet having growing amplitudes.

Here we examine the stability of the compressible mixing layer in order to determine whether it is convectively or absolutely unstable. We have shown (Jackson and Grosch, 1989b) that the qualitative stability characteristics of a compressible mixing layer are independent of the detailed shape of the velocity profile. Therefore in this study we choose to approximate the mean velocity by a hyperbolic tangent profile and to calculate the temperature profile from the Crocco relation. Thus

$$
\begin{gathered}
U=\frac{1}{2}\left[1+\beta_{U}+\left(1-\beta_{U}\right) \tanh (\eta)\right], \\
T=1-\left[\frac{1-\beta_{T}}{1-\beta_{U}}\right](1-U)+\frac{\gamma-1}{2} M^{2}(1-U)\left(U-\beta_{U}\right) .
\end{gathered}
$$

All quantities have been nondimensionalized by the values of the variables in the fast stream, taken here to be at $+\infty$. Here $\beta_{U}$ is the ratio of the speed of the slow stream to that of the fast stream, $\beta_{T}$ is the ratio of the temperature in the slow stream to that of the fast stream, $M$ is the Mach number of the fast stream, $\gamma$ is the ratio of specific heats of the gas, and $\eta$ is the similarity variable in terms of the downstream distance and the Howarth-Dorodnitzyn variable. Note that

$$
-1<\beta_{U}<1, \quad \text { and } \quad \beta_{T}>0 \text {. }
$$

It is well known that the instabilities of compressible free shear flows are predominantly inviscid, except at very low Reynolds numbers. Consequently, we considered only the case of inviscid instability. The formulation and the method of solution of the linearized instability problem for a compressible mixing layer was given by Jackson and Grosch (1989a). The eigenvalue is a zero of the characteristic equation relating the wavenumber $\alpha$ and the frequency $\omega$ at fixed Mach number. Since $\alpha(\omega)$ has a square root branch point singularity at a zero of the complex group velocity $\partial \omega / \partial \alpha$, transition from convective to absolute instability occurs when the zero lies on the real $\omega$ axis. We therefore choose $\omega$ to be real, $\alpha$ to be complex, and carried out a numerical search for a zero of $\partial \omega / \partial \alpha$. In these calculations we took $\gamma=1.4$ and varied $\beta_{U}, \beta_{T}$, and $M$.

Figure 1 shows results for zero Mach number. The locus of the branch point position separating the regions of absolute and convective instability in the $\beta_{U}-\beta_{T}$ plane is 
shown. In the region to the left of this curve, the flow is absolutely unstable, otherwise the flow is convectively unstable. For $\beta_{T}=1, \beta_{U}=-0.136$ which is the value found by Huerre and Monkewitz (1985). Note that increasing $\beta_{T}$ requires a substantial decrease in $\beta_{U}$ to make the flow absolutely unstable. On the other hand, for a fixed negative $\beta_{U}$, cooling the gas of the slow stream can cause the flow to change from convectively to absolutely unstable. When $\beta_{T}$ is small, the equations become stiff and for this reason we were not able to carry out calculations for $\beta_{T}<0.15$. Figure 2 shows related results. The curves separating the regions of absolute and convective instability in the $\beta_{U}-M$ plane is shown for three different values of $\beta_{T}$. In the region below each curve, the flow is convectively unstable and above the curve it is absolutely unstable. Consistent with the results of Figure 1, increasing $\beta_{T}$ causes a shift in the curve to more negative values of $\beta_{U}$ at any Mach number. A consistent trend is apparent for all values of $\beta_{T}$; as the Mach number increases the amount of backflow necessary to cause absolute instability increases.

The results presented in the above figures shows that, for the standard co-flowing mixing layer, the flow is convectively unstable. This has been shown only for subsonic disturbances. Because supersonic disturbances have a very different behavior at either $+\infty$ or $-\infty$, substantial modifications to the Green's function is required. Flow control of the convectively unstable compressible mixing layer can now be achieved by forcing at the frequency of the most unstable mode obtained from spatial stability theory. However, Morkovin (1988) notes that the criterion described above for absolute/convective instabilities is strictly valid only for small disturbance linearized parallel flows. Nonparallel and nonlinear effects can alter these results.

During the course of this study, we became aware of the work of Pavithran and Redekopp (1989). In their work they also examined the absolute/convective instability of a compressible mixing layer. The velocity profile was given by a hyperbolic tangent, as in this study, but the temperature profile is not given by the Crocco relation. Instead, their temperature equation is equivalent to that obtained from (2.2) by dropping the Mach squared term. Despite the fact that this is not strictly correct, it yields a good numerical approximation over the range of Mach numbers considered. Our results differ from those of Pavithran and Redekopp by at most $10 \%$.

3. The Convective Mach Number. The motivation for this study is implicitly contained in the results obtained from our study of the stability of the compressible mixing layer (Jackson and Grosch, 1989b). In this study we took $\beta_{U}=0$, used various values of $\beta_{T}$, varied the Mach number from 0 to 7 , and used a number of thermodynamic models with different values of the Prandtl number and different viscosity-temperature relations. In all cases, we found that the maximum growth rates of the disturbances exhibited a similar dependence on Mach number. As the Mach number was increased from zero the maximum growth rate decreased by a factor of 5 to 10 up to $M_{*}$, the Mach number at which the sonic speeds of the two streams are equal. For Mach numbers greater than $M_{*}$ the maximum growth rates level off. This suggests that $M_{*}$ is the proper Mach number scaling for this stability problem. Thus, a convective Mach number can be defined as 


$$
M_{c}=M / M_{*} .
$$

With this definition, growth rates decrease for $M_{c}<1$, while they level off for $M_{c}>1$.

Motivated by this observation, we derive $M_{*}$ for a multi-species gas as a function of the density, temperature, and velocity ratios of the streams and of the thermodynamics. In section 3.1 we formulate the problem, and in section 3.2 the results are given.

3.1. Formulation of the Problem. The problem considered here is that of the inviscid spatial stability of a steady two dimensional compressible mixing layer which lies between two streams of $\mathrm{N}$ multi-species gases with different speeds and temperatures. We first consider the case of two dimensional disturbances, and then generalize our results to that of three dimensional disturbances. The nondimensional inviscid equations governing this flow are (Williams, 1985)

$$
\begin{aligned}
& \rho_{t}+(\rho u)_{x}+(\rho v)_{y}=0, \\
& \rho\left(u_{t}+u u_{x}+v u_{y}\right)+C_{1} P_{x}=0, \\
& \rho\left(v_{t}+u v_{x}+v v_{y}\right)+C_{1} P_{y}=0, \\
& \rho\left(H_{t}+u H_{x}+v H_{y}\right)-\left(P_{t}+u P_{x}+v P_{y}\right)=0, \\
& \rho\left(F_{j, l}+u F_{j, x}+v F_{j, y}\right)=0, \quad j=1,2, \ldots N, \\
& P=\rho T\left[\frac{\sum_{j=1}^{N}\left(F_{j} / W_{j}\right)}{\sum_{j=1}^{N}\left(F_{j, \infty} / W_{j}\right)}\right]
\end{aligned}
$$

where $\rho$ is the density, $u$ the velocity component in the $x$ direction, $v$ the velocity component in the $y$ direction, $\mathrm{T}$ the temperature, $\mathrm{P}$ the pressure, $F_{j}$ the mass fraction of species $\mathrm{j}$ with molecular weight $W_{j}$, and $\mathrm{H}$ the mixture enthalpy defined by

$$
H=\sum_{j=1}^{N} H_{j} F_{j} / H_{\infty}
$$

where $H_{j}$ is the enthalpy of species $\mathrm{j}$

$$
H_{j}=H_{j}^{o} / H_{\infty}+\int_{T^{o}}^{T} C_{p, j}(T) d T .
$$


Here $C_{p, j}$ is the dimensionless specific heat at constant pressure for each species and the subscript $\infty$ indicates those quantities that are to be measured in the free stream at $y=+\infty$. Finally, the constant $C_{1}$ appearing above is given as

$$
C_{1}=\frac{P_{\infty}}{\rho_{\infty} u_{\infty}^{2}}
$$

The equations were nondimensionalized by selecting the free stream values $\rho_{\infty}, u_{\infty}$, $T_{\infty}, P_{\infty}, H_{\infty}$, at $y=+\infty$ for the density, velocity components, temperature, pressure, and enthalpy, respectively. Lengths are referred to $\mathrm{L}$, some characteristic length scale of the mean flow, while the time scale is the ratio of the length and speed scales. In writing down these equations the only assumption made is that all body forces are neglected.

We assume that the mean flow is given and satisfies the boundary conditions,

$$
\begin{gathered}
u=\rho=T=P=1, \quad \text { at } y=+\infty, \\
u=\beta_{U}, \rho=\beta_{\rho}, \quad T=\beta_{T}, P=1 \quad \text { at } y=-\infty,
\end{gathered}
$$

where $\beta_{U}, \beta_{\rho}$, and $\beta_{T}$ are the ratios of the velocity, density, and temperature, respectively, in the stream at $y=-\infty$ to that of the stream at $y=+\infty$. Note that $\beta_{\mathrm{p}}$ is related to $\beta_{T}$ through the identity

$$
1=\beta_{\rho} \beta_{T}\left[\frac{\sum_{j=1}^{N}\left(F_{j,-\infty} / W_{j}\right)}{\sum_{j=1}^{N}\left(F_{j, \infty} / W_{j}\right)}\right]
$$

The stability problem can be formulated independently of the detailed form of the mean flow profiles. The mean flow field is perturbed by introducing two dimensional wave disturbances of the form

$$
\begin{array}{r}
{\left[u, v, P, \rho, T, H, F_{j}\right](x, y, t)=\left[U, 0,1, \rho, T, H, F_{j}\right](y)} \\
+\varepsilon\left[f, \tilde{\alpha} \bar{\phi}, \Pi, r, \xi, h, f_{j}\right](y) e^{i(\tilde{\alpha} x-\omega t)},
\end{array}
$$

where $\varepsilon<<1, \tilde{\alpha}$ is the complex wavenumber and $\tilde{\omega}$ is the frequency which is taken to be real because we are only treating the spatial stability problem. Substituting (3.6) into the inviscid compressible equations (3.2) yields ordinary differential equations for the perturbation amplitudes. To be compatible with the mean flow equations, the perturbation amplitude equations are rewritten in terms of the similarity variable $\eta$, defined by

$$
Y=\int_{0}^{y} \rho d y, \quad \eta=\frac{Y}{2 \sqrt{x}} .
$$


In terms of the similarity variable $\eta$, the linearized equations governing the perturbation amplitudes are,

$$
\begin{gathered}
i(U-c) r+\rho \rho^{\prime} \phi+\rho\left(i f+\rho \phi^{\prime}\right)=0, \\
\rho\left[i(U-c) f+\rho U^{\prime} \phi\right]=-i C_{1} \Pi, \\
i(U-c) \phi=-C_{1} \Pi^{\prime} / \alpha^{2}, \\
\rho\left[i(U-c) h+\rho H^{\prime} \phi\right]=i(U-c) \Pi, \\
i(U-c) f_{j}+\rho F_{j}^{\prime} \phi=0, \quad j=1,2, \ldots N, \\
\Pi=\sum_{j=1}^{N}\left[\frac{C_{2}}{W_{j}}\right]\left[\rho T f_{j}+T F_{j} r+\rho F_{j} \xi\right],
\end{gathered}
$$

where

$$
\begin{gathered}
h=\sum_{j=1}^{N}\left[H_{j} f_{j}+F_{j} C_{p, j} \xi\right] / H_{\infty}, \\
C_{2}^{-1}=\sum_{j=1}^{N} F_{j, \infty} / W_{j},
\end{gathered}
$$

and

$$
\tilde{\omega}=\omega / 2 \sqrt{x}, \quad \tilde{\alpha}=\alpha / 2 \sqrt{x}, \quad \tilde{\phi}=2 \sqrt{x} \phi .
$$

Here, $\mathrm{c}$ is the complex wave speed

$$
c=\omega / \alpha .
$$

Since $\alpha$ is complex, the real part of $\alpha$ is the wave number in the $x$ direction, while the imaginary part of $\alpha$ indicates whether the disturbance is amplified, neutral, or damped depending on whether $\alpha_{i}$ is negative, zero, or positive, assuming positive group velocity. The phase speed, $c_{p h}$, is given by $\omega / \alpha_{r}$. If $\alpha_{i}$ is zero, $c=c_{N}$ is the phase speed of a neutral mode. Finally, primes indicate differentiation with respect to the similarity variable $\eta$.

From this set it straightforward to derive a single equation governing $\Pi$, given by

$$
\Pi^{\prime \prime}-\left[\frac{2 U^{\prime}}{U-c}\right] \Pi^{\prime}+\left[\left(\frac{\rho^{\prime}}{\rho}\right)+\left[\frac{G_{1}^{\prime}}{G_{1}}\right]+\left[\frac{H^{\prime}-G_{3}}{G_{2} T}\right]\right] \Pi^{\prime}
$$




$$
-\left[\frac{\alpha^{2}}{\rho^{2} T}\right]\left[T-\left(\frac{(U-c)^{2}}{C_{1}}\right)\left[\frac{1}{C_{2} G_{1}}-\frac{1}{G_{2}}\right)\right] \Pi=0,
$$

where

$$
\begin{gathered}
G_{1}(\eta)=\sum_{j=1}^{N}\left[F_{j} / W_{j}\right], \\
G_{2}(\eta)=\sum_{j=1}^{N}\left[F_{j} C_{p, j} / H_{\infty}\right], \\
G_{3}(\eta)=\sum_{j=1}^{N}\left[H_{j} F_{j}^{\prime} / H_{\infty}\right] .
\end{gathered}
$$

For a single species gas and constant specific heat, (3.11) reduces to that of Jackson and Grosch (1989a).

The boundary conditions for $\Pi$ are obtained by considering the limiting form of equation (3.11) as $\eta \rightarrow \pm \infty$. The solution to (3.11) is of the form

$$
\Pi \rightarrow \exp \left( \pm \Omega_{ \pm} \eta\right),
$$

where

$$
\begin{gathered}
\Omega_{+}^{2}=\alpha^{2}\left[1-\left(\frac{(1-c)^{2}}{C_{1}}\right)\left[1-\frac{1}{G_{2, \infty}}\right)\right], \\
\Omega_{-}^{2}=\left[\frac{\alpha^{2}}{\beta_{\rho}^{2} \beta_{T}}\right]\left[\beta_{T}-\left(\frac{\left(\beta_{U}-c\right)^{2}}{C_{1}}\right)\left[\frac{1}{C_{2} G_{1,-\infty}}-\frac{1}{G_{2,-\infty}}\right)\right] .
\end{gathered}
$$

We define $c_{ \pm}$to be the values of the phase speed for which $\Omega_{ \pm}^{2}$ vanishes. Thus,

$$
\begin{gathered}
c_{+}=1-\left[\frac{C_{1}}{1-\frac{1}{G_{2, \infty}}}\right]^{1 / 2}, \\
c_{-}=\beta_{U}+\sqrt{\beta_{T}}\left[\frac{C_{1}}{\frac{1}{C_{2} G_{1,-\infty}}-\frac{1}{G_{2,-\infty}}}\right]^{1 / 2}
\end{gathered}
$$


Note that $c_{+}$is the phase speed of a sonic disturbance at $\eta=+\infty$ and $c_{-}$is the phase speed of a sonic disturbance at $\eta=-\infty$. If we define the mixture quantities

$$
\begin{gathered}
C_{p}=\sum_{j=1}^{N} F_{j} C_{p, j}, \\
\gamma=\left[\sum_{j=1}^{N} \gamma_{j} F_{j} / W_{j}\right]\left[\sum_{j=1}^{N} F_{j} / W_{j}\right]^{-1},
\end{gathered}
$$

and use the identity for the sound speed

$$
a^{2}=(\gamma-1) C_{p} T=\gamma P / \rho,
$$

then $c_{ \pm}$can be rewritten in terms of the mixture Mach number at $\eta=+\infty$

$$
M=u_{\infty} / a_{\infty},
$$

as follows

$$
\begin{gathered}
c_{+}=1-\frac{1}{M}, \\
c_{-}=\beta_{U}+\frac{1}{M \sqrt{\beta_{\rho} / \beta_{\gamma}}},
\end{gathered}
$$

where

$$
\beta_{\gamma}=\gamma_{-\infty} / \gamma_{\infty}
$$

At

$$
M=M_{*} \equiv \frac{1+\sqrt{\beta_{\gamma} / \beta_{p}}}{1-\beta_{U}}
$$

$c_{ \pm}$are equal. Thus, from (3.1),

$$
M_{c}=\frac{M}{M_{*}}=\frac{M\left(1-\beta_{U}\right)}{1+\sqrt{\beta_{\gamma} / \beta_{\rho}}} .
$$

The nature of the disturbances and the appropriate boundary conditions can now be illustrated by reference to Figure 3, where we plot $c_{ \pm}$versus $M_{c}$. In what follows we assume that $\alpha^{2}{ }_{r}>\alpha^{2}{ }_{i}$. These curves divide the $c_{r}-M_{c}$ plane into four regions, where $c_{r}$ is the real part of c. If a disturbance exists with a $M_{c}$ and $c_{r}$ in region 1 , then $\Omega^{2}{ }_{+}$and $\Omega^{2}$ are both positive, and the disturbance is subsonic at both boundaries. In region 3 , both 
$\Omega^{2}{ }_{+}$and $\Omega^{2}$ - are negative and hence the disturbance is supersonic at both boundaries. In region $2, \Omega^{2}{ }_{+}$is positive and $\Omega^{2}$ is negative, and the disturbance is subsonic at ${ }^{+\infty}$ and supersonic at $-\infty$, and we classify it as a fast mode. Finally, in region $4, \Omega^{2}{ }_{+}$is negative and $\Omega^{2}$ - is positive so the disturbance is supersonic at $+\infty$ and subsonic at $-\infty$, and we classify it as a slow mode.

To complete the stability problem, one can now see that the appropriate boundary condition for either damped or outgoing waves in the moving and stationary streams are, respectively,

$$
\begin{aligned}
& \Pi \rightarrow e^{-\Omega_{+} \eta}, \quad \text { if } c_{r}>c_{+}, \quad \Pi \rightarrow e^{-i \eta \sqrt{-\Omega^{2}}}, \quad \text { if } \quad c_{r}<c_{+}, \\
& \Pi \rightarrow e^{\Omega_{-} \eta}, \quad \text { if } c_{r}<c_{-}, \quad \Pi \rightarrow e^{-i \eta \sqrt{-\Omega^{2}}}, \quad \text { if } \quad c_{r}>c_{-} \text {. }
\end{aligned}
$$

3.2. Results. The effect of the scaling parameter $M_{c}$ can now be analyzed. In our previously reported calculations (Jackson and Grosch, 1989b) we considered only a single species gas with $\beta_{U}=0$ and $\beta_{\gamma}=1$. For this case the convective Mach number (3.23) reduces to

$$
M_{c}=\frac{M}{1+\sqrt{\beta_{T}}} \text {. }
$$

In order to present the variation of the maximum growth rates with $M_{c}$ we normalize them by defining the normalized growth rate by

$$
R=\frac{\left(-\alpha_{i}\right)_{M A X}\left(\beta_{T}, M\right)}{\left(-\alpha_{i}\right)_{M A X}\left(\beta_{T}, 0\right)} .
$$

Figure 4 is a plot of $\mathrm{R}$ versus $M_{c}$ for the three thermodynamic models and $\beta_{T}$ of $0.5,1$, and 2. This data is taken from Jackson and Grosch (1989b). It can be seen that with these scalings the data collapses onto essentially a single curve for $M_{c}<1$, and a narrow band for $M_{c}>1$. One should also note that the second unstable modes appear around $M_{c}=1$. This curve is similar to that obtained by Ragab and $\mathrm{Wu}$ (1988) who use Bogdanoff's heuristic definition of the convective Mach number. However, their graph is incomplete and absent of the second mode.

It is instructive at this point to compare our convective Mach number (3.23) to that derived by other authors using hueristic arguments. There are basically three definitions used. These are, in our notaion, that of Bogdanoff (1983)

$$
M_{c}=\frac{M\left(1-\beta_{U}\right)}{\left(1+1 / \sqrt{\beta_{\rho}}\right) \beta_{\gamma}^{1 / 4}},
$$


Papamoschou and Roshko $(1986,1988)$

$$
M_{c}=\frac{M\left(1-\beta_{U}\right)}{1+1 / \sqrt{\beta_{\rho}}},
$$

and Zhuang, Kubota, and Dimotakis (1988)

$$
M_{c}=M\left(1-c_{p h}\right),
$$

where $c_{p h}$ is the phase speed of the most unstable mode from linear stability theory. For a single species gas $\beta_{\gamma}=1$ and our definition of the convective Mach number and that of Bogdanoff and Papamoschou and Roshko are equal. For most multi-species gases $\beta_{\gamma}$ is close to one and all three definitions yield values of the convective Mach number that are about the same. Table 1 contains a comparison of the values of the convective Mach number using the definitions given in the equations (3.23), (3.27), and (3.28), and the data of Papamoschou and Roshko (1988). The definition of (3.29) requires knowledge of the values of the phase speed $c_{p h}$ of the most unstable wave before one can calculate $M_{c}$.

Further understanding of the role of the convective Mach number can be gained by noting $M_{c}=1$ implies that $M=M_{*}$. This is the largest value of the Mach number for which any subsonic instability waves can exist. For larger values of the Mach number there are only supersonic modes which radiate into one or the other stream. The largest value of the convective Mach number for which only subsonic modes can exist is given by

$$
M_{c_{M I N}}=\min \left(M_{+}, M_{-}\right) / M_{*},
$$

where $M_{*}$ is given by (3.22) and $M_{+}$is the value of the Mach number for which $c_{+}$is zero, and $M_{-}$is the value for which $c_{-}$is one. In the intermediate range $M_{C_{M I N}}<M_{c}<1$ both subsonic and supersonic instability waves can exist.

All previous results are for two dimensional disturbances. If three dimensional disturbances were allowed, the only effect would be to replace the Mach number $M$ by the effective Mach number $M \cos (\theta)$, where $\theta$ is the direction of the propagation of the disturbance. Thus, an effective convective Mach number can be defined by

$$
M_{c_{e f f}}=M_{c} \cos (\theta)
$$

with $M_{c}$ given by (3.23). The introduction of the $\cos (\theta)$ factor to produce an effective convective Mach number has been suggested by a number of other authors based on heuristic arguments. Figure 5 contains curves of the normalized maximum growth rate

$$
R=\frac{\left(-\alpha_{i}\right)_{M A X}\left(\beta_{T}, M, \theta\right)}{\left(-\alpha_{i}\right)_{M A X}\left(\beta_{T}, 0,0^{\circ}\right)} .
$$


versus the effective convective Mach number for the Tanh model with $\beta_{T}=1 / 2$ and $\beta_{U}=0$. These curves are based on the numerical calculations of Jackson and Grosch, 1989a. Results are shown for $\theta=0^{\circ}, 20^{\circ}, 40^{\circ}, 60^{\circ}$. For angles of propagation greater than $60^{\circ}$ it was found (Jackson and Grosch, 1989a) that the maximum growth rate begins to decrease with increasing angles of propagation. The data points shown in this figure are taken from the experimential results of Papamoschou and Roshko, 1988. Morkovin (1987) has suggested that three dimensional disturbances dominate the flow field once the effective convective Mach number exceeds one. This insight is borne out by the results shown in this figure.

The above analysis is strictly valid when $\beta_{U}$ is not close to one. If $\beta_{U}$ were taken to be one, then $U=1$ is a solution for the mean velocity profile, but the temperature would still vary throughout the flow field (if $\beta_{T}$ is not equal to one). Any instability present would be a purely thermal one and the physics would be different. Thus decreasing $M_{c}$ by increasing $\beta_{U}$ to one is inconsistent with the formulation of the problem. It appears that the only ways to decrease the convective Mach number is to increase the ratio $\beta_{\gamma} / \beta_{\rho}$ or to increase the angle of propagation of the disturbances.

Finally, the above formulation is also valid for a mixing layer in a channel with a zero streamwise pressure gradient. 


\section{REFERENCES}

Bogdanoff, D.W. 1983 Compressibility Effects in Turbulent Shear Layers. AIAA Journal, 21, 926-927.

Briggs, R.J. 1964 Electron-Stream Interaction with Plasmas. Research Monograph No. 29, MIT Press, Cambridge, Mass.

Gaster, M. 1968 Growth of Disturbances in Both Space and Time. Phys. Fluids, 11, 723-727.

Gaster, M. 1981 On Transition to Turbulence in Boundary Layers. In Transition and Turbulence, R.E. Meyer (ed.), Academic Press, 95-112.

Guirguis, R.H. 1988 Mixing Enhancement in Supersonic Shear Layers: III. Effect of Convective Mach Number. AIAA 88-0701.

Huerre, P. 1987 Spatio-Temporal Instabilities in Closed and Open Flows. In Instabilities and Nonequilibrium Structures, E. Triapegui and D. Villarroel (eds.), D. Reidel Publishing Co., 141-177.

Huerre, P. \& Monkewitz, P.A. 1985 Absolute and Convective Instabilities in Free Shear Layers. J. Fluid Mech., 159, 151-168.

Hultgren, L.S. \& Aggarwal, A.K. 1987 Absolute Instability of the Gaussian Wake Profile. Phys. Fluids, 30, 3383-3387.

Jackson, T.L. \& Grosch, C.E. 1989a Inviscid Spatial Stability of a Compressible Mixing Layer. J. Fluid Mech., in press.

Jackson, T.L. \& Grosch, C.E. 1989b Inviscid Spatial Stability of a Compressible Mixing Layer. Part III. Effect of Thermodynamics. ICASE Report No. 89-32. Also, J. Fluid Mech., submitted.

Leib, S.J. \& Goldstein, M.E. 1986a The Generation of Capillary Instabilities on a Liquid Jet. J. Fluid Mech., 168, 479-500.

Leib, S.J. \& Goldstein, M.E. 1986b Convective and Absolute Instability of a Viscous Liquid Jet. Phys. Fluids, 29, 952-954.

Lebe, S.K. 1989 Direct Numerical Simulation of Compressible Free Shear Layer Flows. AIAA 89-0374.

Monkewitz, P.A. 1988 The Absolute and Convective Nature of Instability in TwoDimensional Wakes at Low Reynolds Numbers. Phys. Fluids, 31, 999-1006.

Morkovin, M.V. 1987 Transition at Hypersonic Speeds. ICASE Interim Report No. 1. 
Morkovin, M.V. 1988 Recent Insights into Instability and Transition to Turbulence in Open-Flow Systems. AIAA 88-3675.

Mukunda, H.S., Sekar, B., Carpenter, M., Drummond, J.P., \& Kumar, A. 1989 Studies in Direct Simulations of High Speed Mixing Layers. NASA TP, to appear.

Papamoschou, D. \& Roshko, A. 1986 Observations of Supersonic Free Shear Layers. AIAA 86-0162.

Papamoschou, D. \& Roshko, A. 1988 The Compressible Turbulent Shear Layer: an Experimental Study. J. Fluid Mech., 197, 453-477.

Pavithran, S. \& Redekopp, L.G. 1989 The Absolute-Convective Transition in Subsonic Mixing Layers. Phys. Fluids, in press.

Sandham, N. \& Reynolds, W. 1989 The Compressible Mixing Layer: Linear Theory and Direct Simulation. AIAA 89-0371.

Williams, F.A. 1985 Combustion Theory, 2nd Ed., The Benjamin/Cummings Pub. Co., Menlo Park, CA.

Zhuang, M., Kubota, T. \& Dimotakis, P.E. 1988 On the Instability of Inviscid, Compressible Free Shear Layers. AIAA 88-3538. 


\begin{tabular}{|cccccccc|}
\hline Gas 1 & Gas 2 & M & $\beta_{\rho}$ & $\beta_{u}$ & $M_{c}$ & $M_{c}$ & $M_{c}$ \\
& & & & & $(3.23)$ & $(3.27)$ & $(3.28)$ \\
& & & & & & & \\
& & & & & & & \\
$\mathrm{Ar}$ & $\mathrm{Ar}$ & 3.4 & 0.43 & 0.81 & 0.26 & 0.26 & 0.26 \\
$N_{2}$ & $N_{2}$ & 3.1 & 0.54 & 0.74 & 0.34 & 0.34 & 0.33 \\
$N_{2}$ & $\mathrm{Ar}$ & 2.8 & 1.80 & 0.75 & 0.39 & 0.37 & 0.39 \\
$N_{2}$ & $\mathrm{Ar}$ & 3.2 & 1.20 & 0.67 & 0.53 & 0.53 & 0.55 \\
$\mathrm{He}$ & $N_{2}$ & 1.7 & 9.20 & 0.52 & 0.60 & 0.61 & 0.64 \\
$\mathrm{Ar}$ & $\mathrm{Ar}$ & 3.1 & 0.24 & 0.13 & 0.89 & 0.89 & 0.89 \\
$\mathrm{He}$ & $N_{2}$ & 2.6 & 5.50 & 0.42 & 1.03 & 1.01 & 1.05 \\
$\mathrm{He}$ & $N_{2}$ & 3.4 & 2.20 & 0.29 & 1.39 & 1.38 & 1.44 \\
$\mathrm{He}$ & $\mathrm{Ar}$ & 3.1 & 2.40 & 0.04 & 1.81 & 1.81 & 1.81 \\
& & & & & & & \\
\hline
\end{tabular}

Table 1. Comparison of the convective Mach number values using the definitions of this paper (3.23), Bogdanoff (3.27), and Papamoschou and Roshko (3.28) and the data of Papamoschou and Roshko, 1988. 


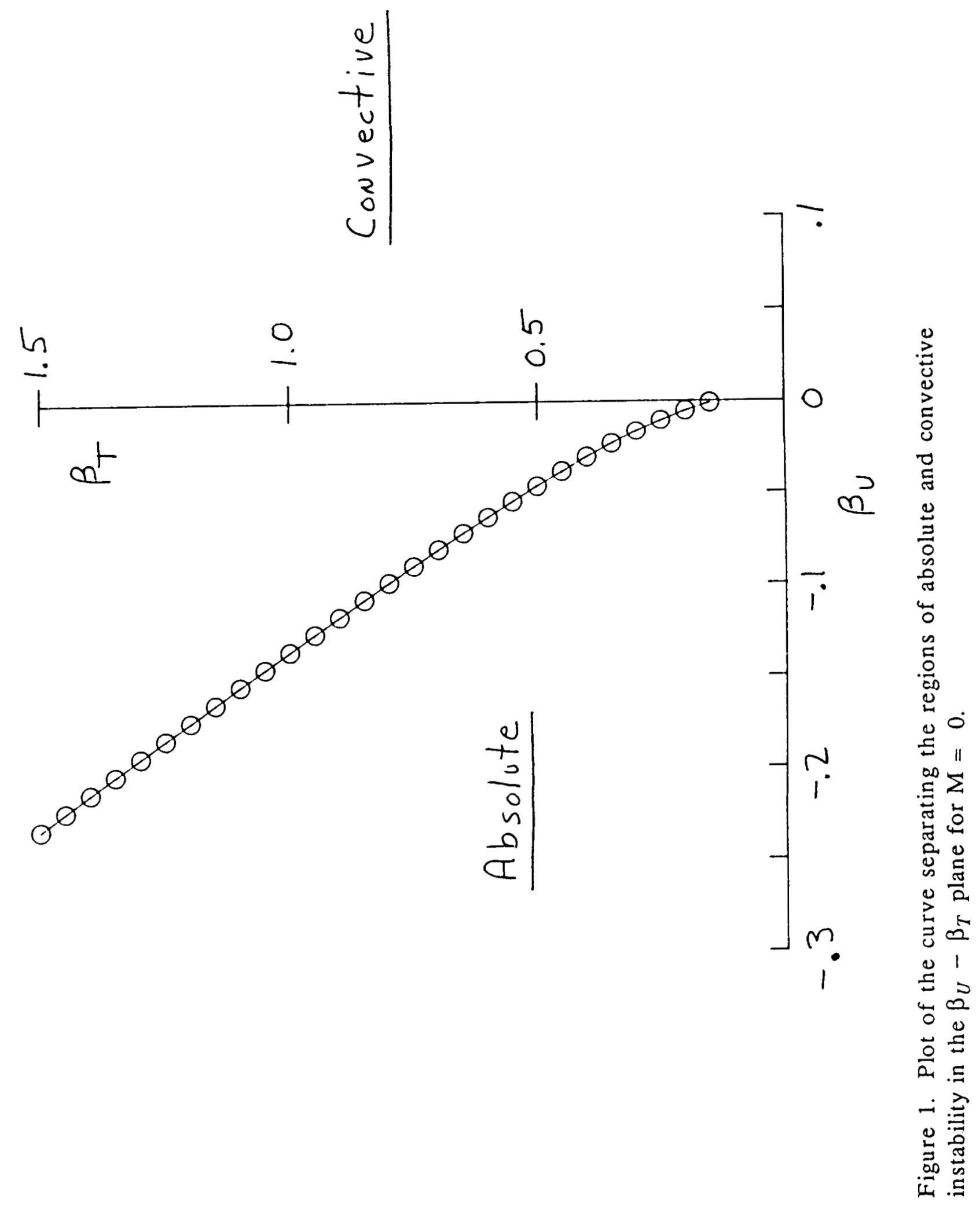



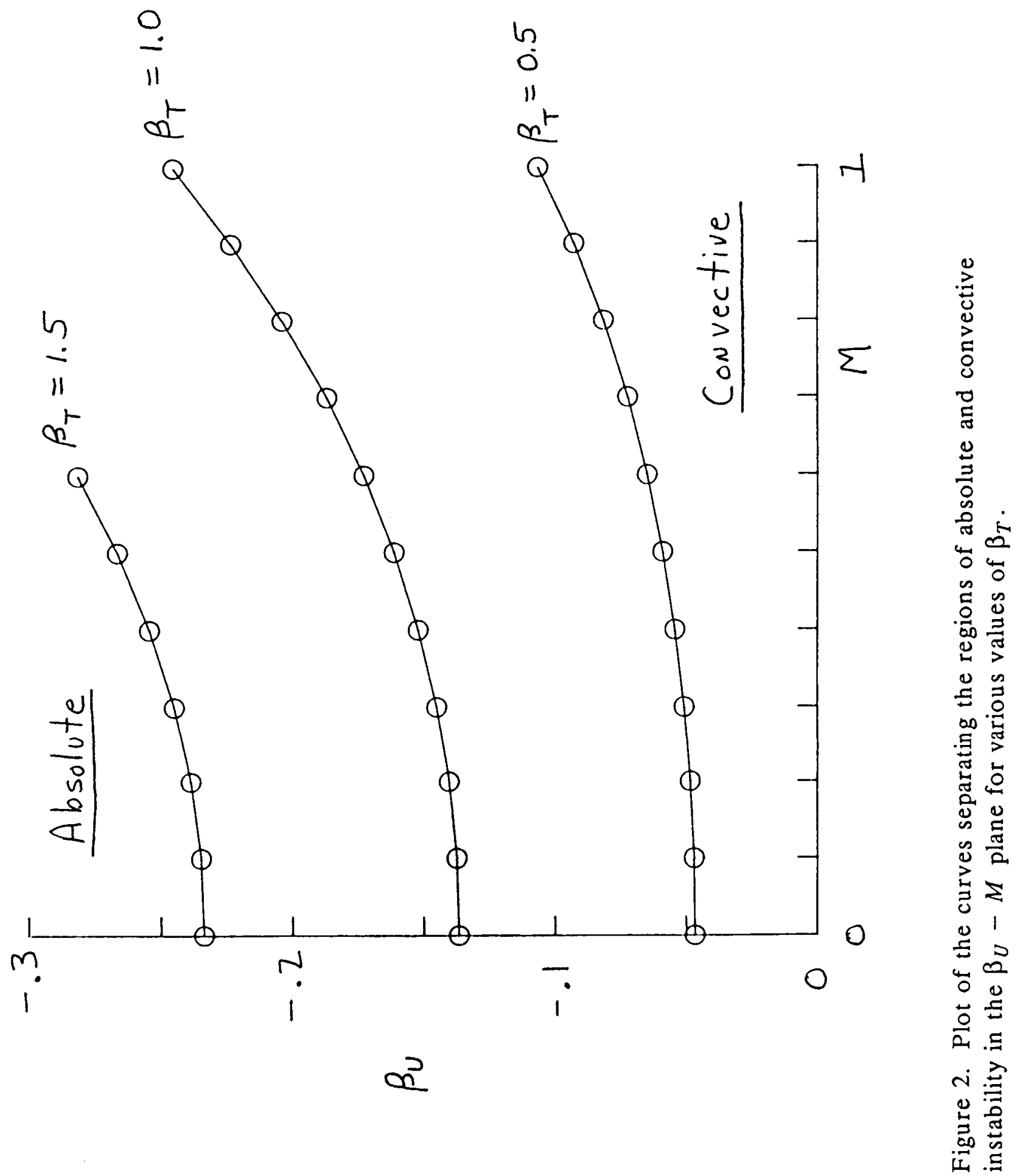


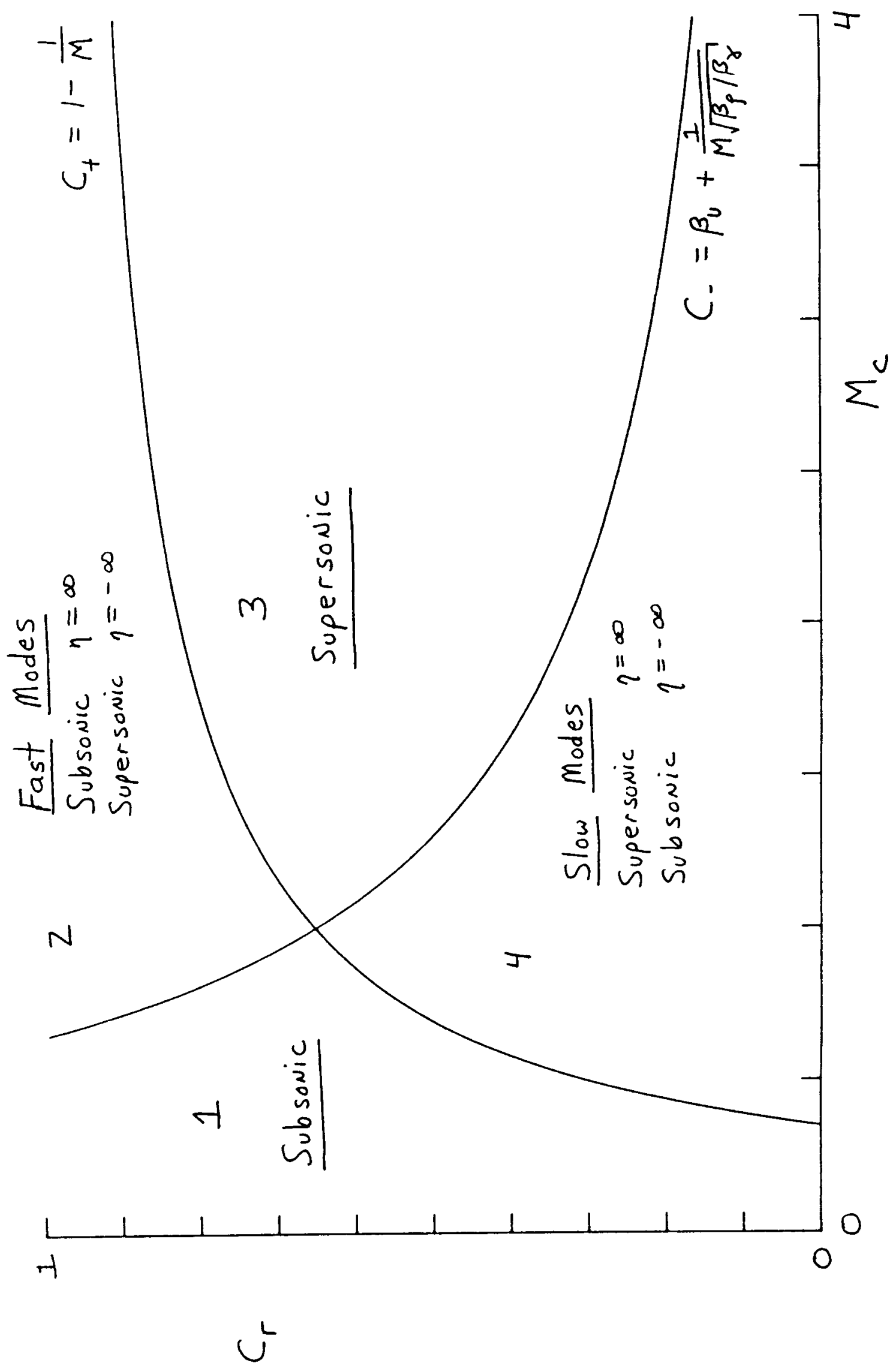




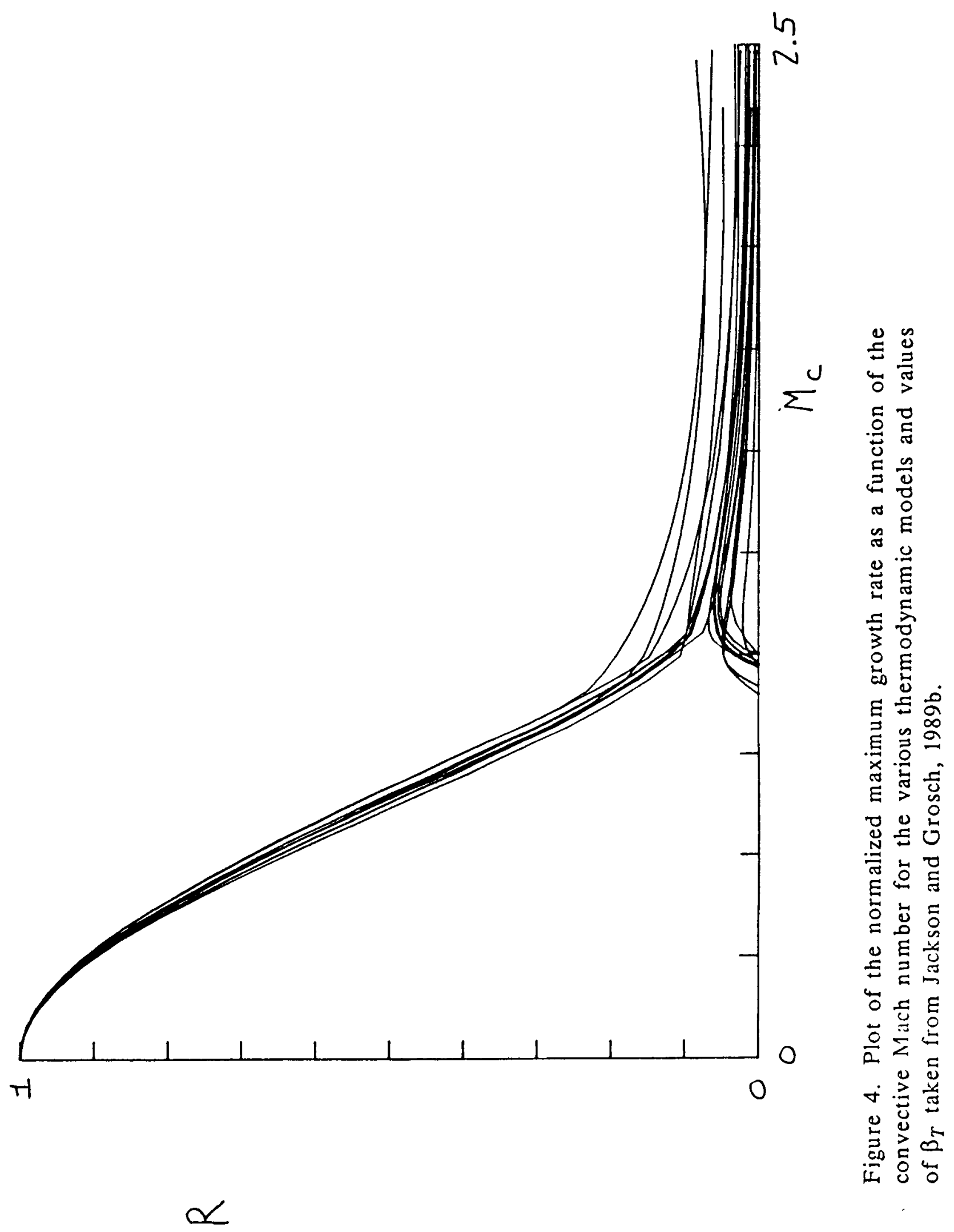




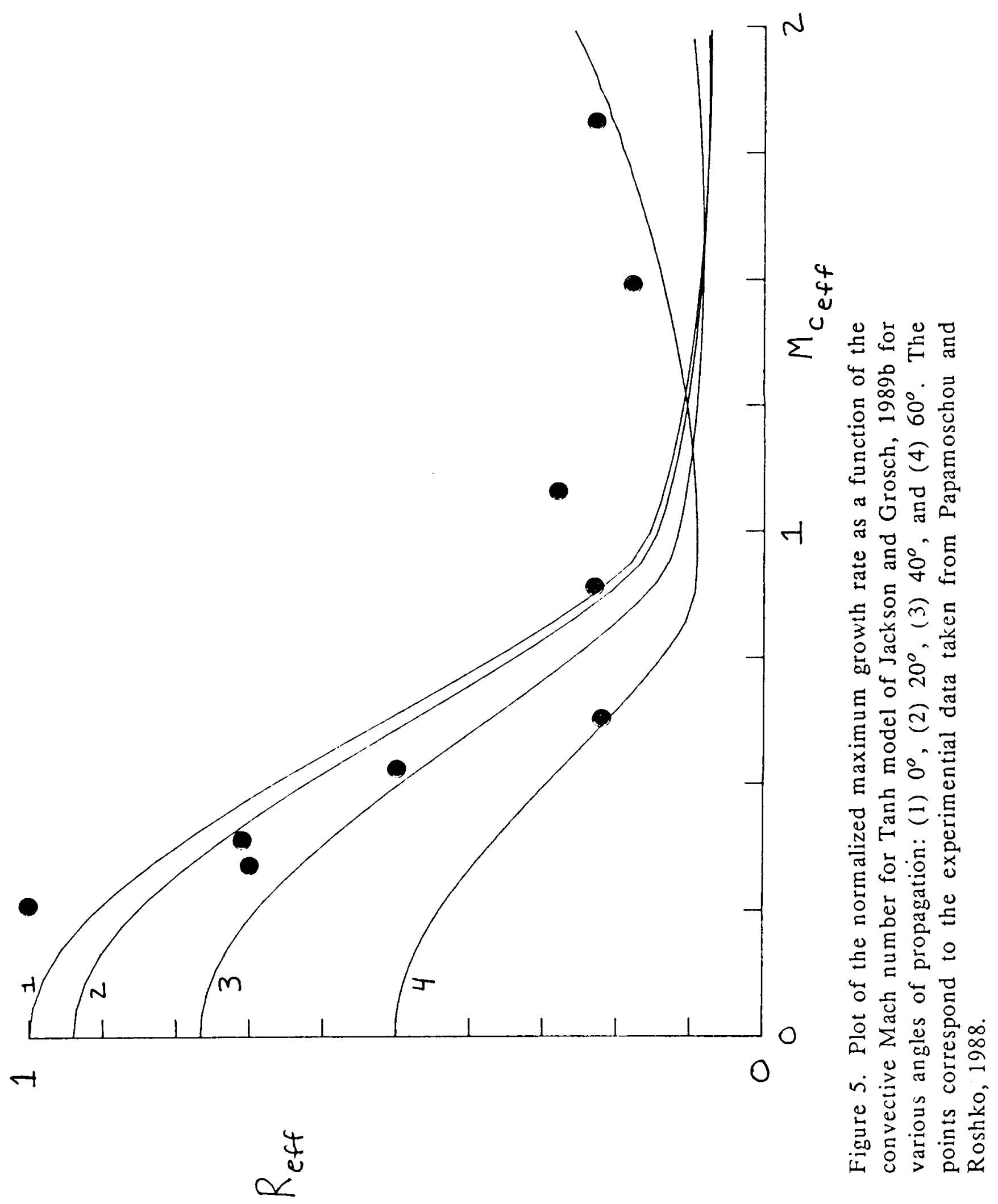




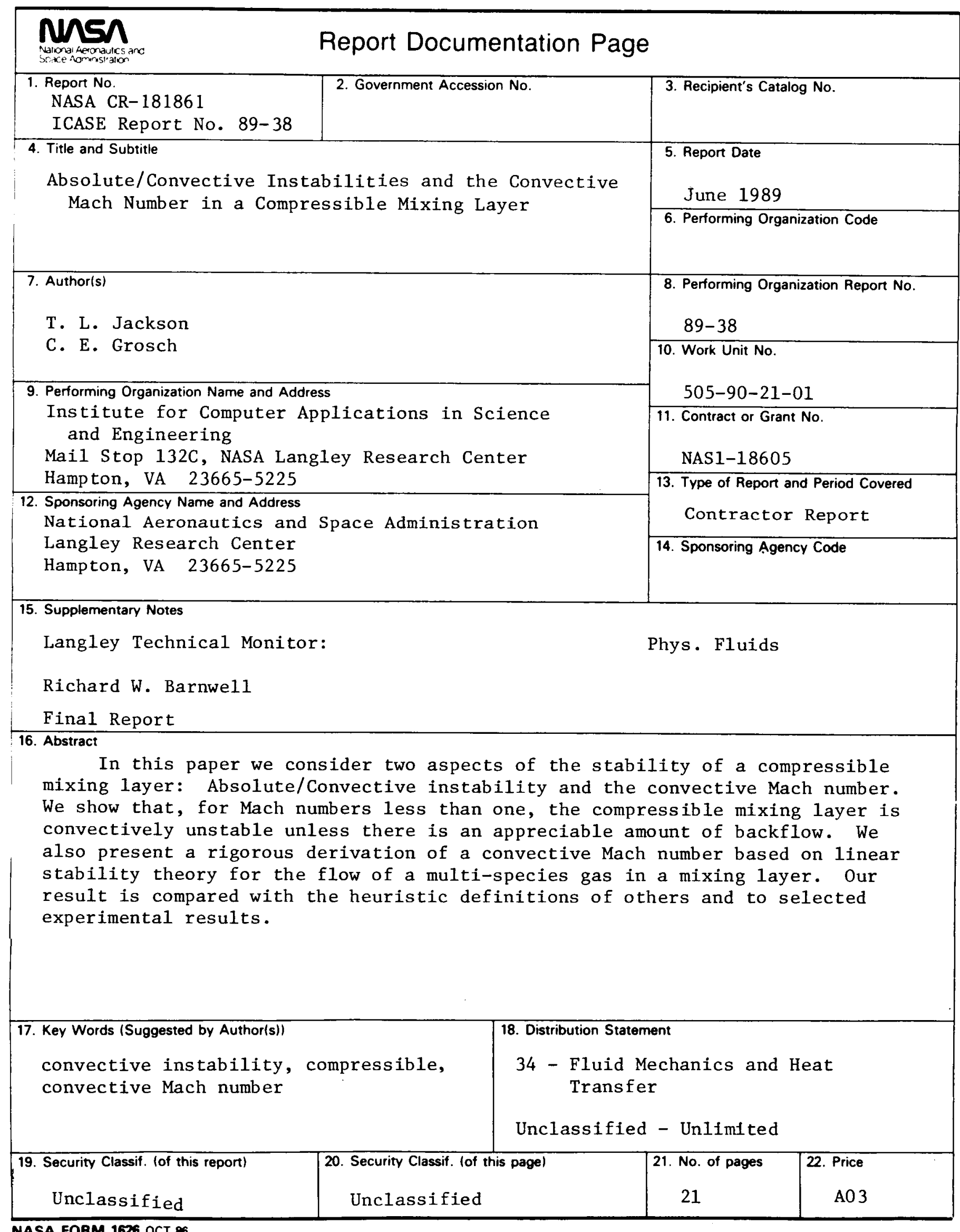

